\title{
A dynamically consistent analysis of circulation and transports in the southwestern Weddell Sea
}

\author{
M. Yaremchuk ${ }^{1,2}$, D. Nechaev ${ }^{1,2}$, J. Schroter ${ }^{1}$ E. Fahrbach ${ }^{1}$ \\ ${ }^{1}$ Alfred-Wegener-Institute for Polar and Marine Research, Am Handelshafen 12, D-27570 Bremerhaven, Germany \\ ${ }^{2}$ Shirshov Institute of Oceanography, Krasikova 23, 117218 Moscow, Russia
}

Received: 22 January 1997 / Revised: 27 February 1998 / Accepted: 10 March 1998

\begin{abstract}
An inverse model is applied for the analysis of hydrographic and current meter data collected on the repeat WOCE section SR4 in the Weddell Sea in 19891992. The section crosses the Weddell Sea cyclonic gyre from Kapp Norvegia to the northern end of the Antarctic Peninsula. The concepts of geostrophy, conservation of planetary vorticity and hydrostatics are combined with advective balances of active and passive properties to provide a dynamically consistent circulation pattern. Our variational assimilation scheme allows the calculation of three-dimensional velocities in the section plane. Current speeds are small except along the coasts where they reach up to $12 \mathrm{~cm} / \mathrm{s}$. We diagnose a gyre transport of 34 Sverdrup which is associated with a poleward heat transport of $28 \times 10^{12} \mathrm{~W}$ corresponding to an average heat flux of $15 \mathrm{Wm}^{-2}$ in the Weddell Sea south of the transect. This exceeds the estimated local flux on the transect of $2 \mathrm{Wm}^{-2}$. As the transect is located mostly in the open ocean, we conclude that the shelf areas contribute significantly to the ocean-atmosphere exchange and are consequently key areas for the contribution of the Weddell Sea to global ocean ventilation. Conversion of water masses occuring south of the section transform $6.6 \pm 1.1 \mathrm{~Sv}$ of the inflowing warm deep water into approximately equal amounts of Weddell Sea deep water and Weddell Sea bottom water. The volume transport of surface water equals in the inand outflow. This means that almost all newly formed surface water is involved in the deep and bottom water formation. Comparison with the results obtained by pure velocity interpolation combined with a hydrographic data subset indicates major differences in the derived salt transports and the water mass conversion of the surface water. The differences can be explained by deviations in the structure of the upper ocean currents to which shelf areas contribute significantly. Additionally a rigorous variance analysis is performed. When only hydrographic data are used for the inversion both the
\end{abstract}

Correspondence to: D. Nechaev gyre transport and the poleward heat transport are substantially lower. They amount to less than $40 \%$ of our best estimate while the standard deviations of both quantities are $6.5 \mathrm{~Sv}$ and $37 \times 10^{12} \mathrm{~W}$, respectively. With the help of long-term current meter measurements these errors can be reduced to $2 \mathrm{~Sv}$ and $8 \times 10^{12} \mathrm{~W}$. Our result underlines the importance of velocity data or equivalent information that helps to estimate the absolute velocities.

Key words. Oceanography: General (Arctic and antarctic oceanography) - Oceanography: Physical (General circulation; Hydrography)

\section{Introduction}

In recent years the Southern Ocean and the Antarctic coastal seas have been the subject of intense studies. Physical processes in these remote areas of the World Ocean strongly affect both global atmospheric and oceanic dynamics. The Weddell Sea is usually considered as the Antarctic coastal basin with the largest impact on the global thermohaline circulation.

The present study is aimed at extracting a "steady state" component of the circulation in the Weddell Sea from hydrographic and current meter data obtained between 1989 and 1992. We assume that there exists a meaningful steady state obtained as a temporal average over the measurement period and deviations from that state on time scales up to a few years are small compared to the amplitude of the steady state field itself.

General hydrographic features of the Weddell Sea are assumed to be well established qualitatively e.g. Gill (1973) and Orsi et al. (1993), whereas quantitative estimates of transports, fluxes and water mass formation rates demonstrate a large scatter in the literature. As an example, the different estimates of the Weddell Gyre 
transport vary within the range between 10 and $96 \mathrm{~Sv}$ (see Carmack and Forster, 1975; Gordon et al., 1981; Fahrbach et al., 1991; Olbers and Wübber, 1991). The latest transport estimates based on long-term current meter records and intense field studies in the Weddell Sea give a value of $30 \mathrm{~Sv}$ (Fahrbach et al., 1994b).

Considering confidence limits for the estimates of heat fluxes and water mass formation rates, the situation may be even worse. These estimates are usually deduced indirectly as the difference of large numbers of somewhat uncertain values of transports. As a consequence, estimates of the formation rate of the Weddell Sea bottom water vary within the range of 0.8-9 Sv (see Gill, 1973; Weiss et al., 1979; Ivanov, 1981; Fahrbach et al., 1994c; Fahrbach et al., 1995).

In order to reduce the uncertainties, an analysis was carried out which supplied statistically and dynamically consistent results. The consistency can be used to derive error bars that take into account the full covariance matrix of the solution.

The method used in the present work can also be viewed as a kind of generalization of the well-known inverse methods, proposed by Wunsch (1994) and Stommel and Schott (1977) who reconstruct large-scale currents utilizing approximately the same set of dynamical constraints as we do. The principal difference is that dynamics in the $\beta$-spiral method of Stommel and Schott (1997) is satisfied by the least squares method while density measurements are considered to be perfect, whereas Wunsch (1994) seeks for an exact fit to dynamics by varying the measured density field. In our approach both dynamics and data are treated with pre-defined degrees of uncertainty, which are discussed in Sect. 3.

In our study we assemble a combination of different types of data into a quantitative description of the Weddell Sea state on the section plane from Kapp Norvegia to the northern tip of the Antarctic Peninsula. The assimilation scheme underlying our analysis is based on the simple dynamical and statistical principles which constrain the assimilated fields. A straightforward but time-consuming calculation allows to estimate the error bars for any feature of the ocean state. The constraints are written as a circulation model in finite difference form. Steady state is assumed and we solve for the most probable solution. The method used here has been applied previously with simpler statistical principles and cruder error analysis to WOCE section S4 by Nechaev and Yaremchuk (1995).

Part of the data sets under consideration have already been analyzed by different authors, e.g. Fahrbach et al. (1994) and Nechaev et al. (1995). Our emphasis is placed on the analysis of the "steady state component" derived from the combination of various types of data from several years. Another important task was to identify the role of in situ velocity measurements in revealing the large-scale circulation pattern and the effect of missing measurements of upper ocean currents due to the requirements of the mooring techniques. The results presented contribute to the understanding of the Weddell Gyre circulation and water mass conversion.
This study is organized as follows. After a brief description of the data in Sect. 2, we present the parameters of the inverse model in Sect. 3, paying special attention to the definitions of prior covariances and to the description of the posterior error analysis scheme. In Sect. 4, results are presented for different data sets and for their combinations. The discussion of the solution accompanied by rigorous variance analysis is followed by the final conclusions.

\section{The data}

\subsection{Tracers}

Our analysis deals with the data set which has been collected during the course of a multiyear program, the Weddell Gyre Study, a contribution to the World Ocean Circulation Experiment. Measurements were taken along repeat section SR4 which connects Kapp Norvegia, the center of the Weddell Gyre and the northern tip of the Antarctic Peninsula (Fig. 1). Due to the effect of the ice coverage on the ship's course, deviations of the section of up to $30 \mathrm{~km}$ occurred. Hydrographic surveys were made in September-October, 1989, NovemberDecember, 1990 and December 1992-January 1993 (see Fig. 1 and Fahrbach, 1992, 1994; Goeyens et al., 1991 Augstein et al., 1991). In total 150 stations were processed.

Figure 2a-2c depicts vertical sections of in situ temperature $T$ obtained by linear interpolation from station locations onto the neighboring regular grid points. The sections have been performed consecutively in late winter, spring and early summer conditions. Consequently the data contain a certain bias towards "spring state". However as seasonal variations dominate only in the upper layer and some parts of the boundary currents, we expect only minor effects of that bias on the derived integral quantities. Actually, it will be shown later that variations of the integral features from section to section lie within the error bars derived under the assumption of the existence of a meaningful steady state.

As already mentioned the sections shown in Fig. 2 were made along slightly different routes (see Fig. 1), and differences occur in bottom topography near the continental slopes. The variations in track positions give valuable information about the oceanic variability on scales of $10-100 \mathrm{~km}$. These scales are poorly resolved by station spacing in the middle of the gyre and they are filtered out in the final solution. A gradual warming visible in Fig. 2 is most evident in the increase of the area occupied by the warm deep water (WDW) with temperatures above $0.6{ }^{\circ} \mathrm{C}$. The mean temperature of the water on the section rose from $-0.127^{\circ} \mathrm{C}$ in 1989 to $-0.105^{\circ} \mathrm{C}$ in 1990 and to $-0.092{ }^{\circ} \mathrm{C}$ in 1992 . This change reflects significant interannual variability in the data underlining the need for long-term arrays to obtain a reliable estimate of the oceanic state in the area.

A composite hydrographic data set was obtained by projecting data from the 1989 and 1990 stations onto the 


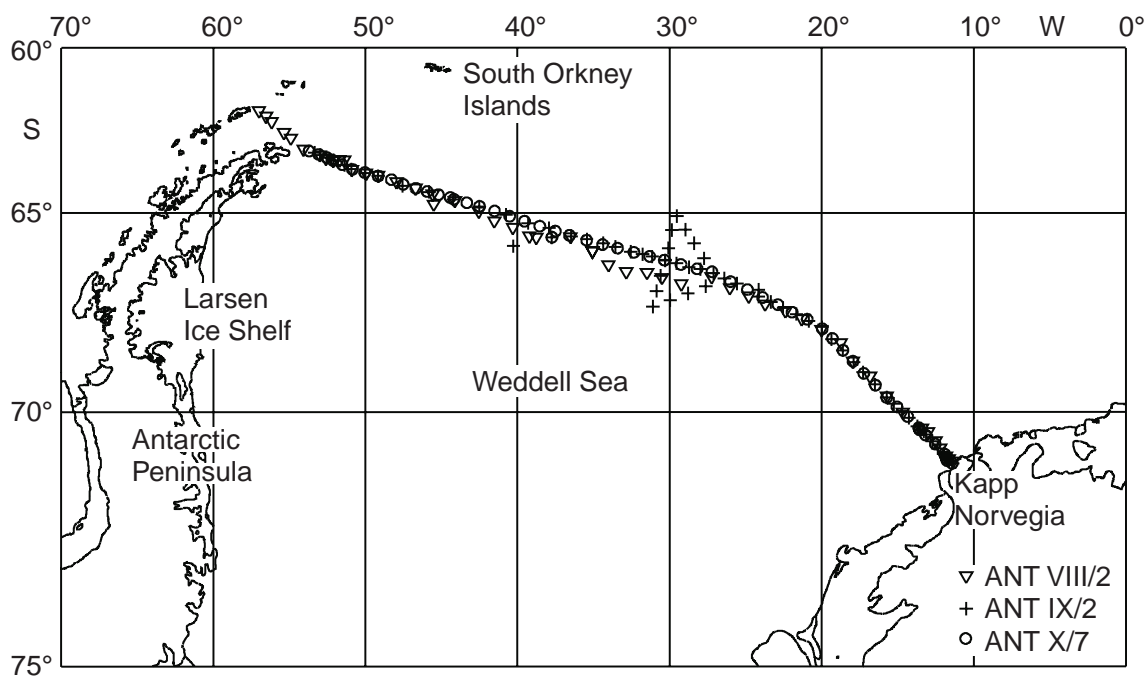

Fig. 1. CTD/rosette stations on the section across the Weddell Sea sampled during the three surveys in 1989 (triangles), 1990 (crosses) and 1992 (circles)
1992 section (Fig. 2d). In the regions over steep topographic features (continental slopes) 1989 and 1990 stations were displaced to 1992 line along the contours of constant depth, while in the other regions a normal projection onto the 1992 line was used.

On all three sections bottle data of oxygen, silicate, nitrate and phosphate were taken. Additionally we used ammonium data obtained in 1989, 1990 and the results of $\mathrm{CO}_{2}$ measurements in 1992. Those data contribute to the general circulation pattern in two ways. Firstly, there are strong cross-correlations between different parameters, effectively decreasing the number of degrees of freedom for the assimilation problem. Secondly, they provide additional information for the velocity field,
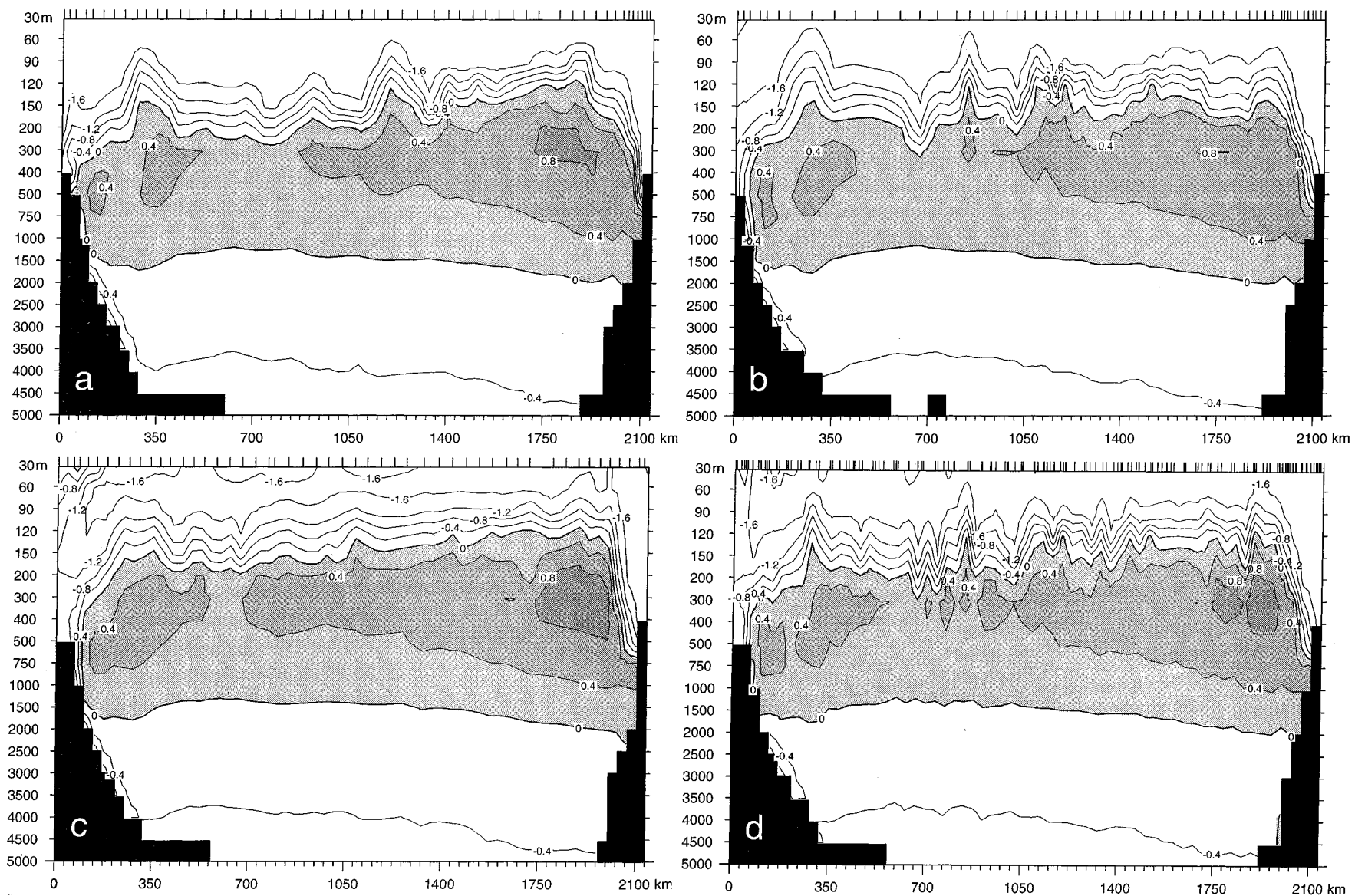

Fig. 2a-d. Temperature fields obtained by linear interpolation of the data measured a in 1989 b 1990 and c $1992-1993$ onto the model grid; d shows the composite data set. Station locations are shown by markers the upper part of the maps 
which is constrained by advective equations. The total number of data from different parameters used in the analysis amounts to 25470 .

\subsection{Velocity data}

Along the section, 45 current meter records were taken over more than one year each. These measurements were concentrated mostly in the boundary currents and near the bottom (Fig. 3).

The current meter data are projected onto the grid points neighboring the locations of the current meters. Means of the along track and cross track velocity components $u$ and $v$ are derived. A detailed description of the velocity data have been given previously (Fahrbach et al., 1994). Figure 3 is adapted from Fahrbach et al. (1994) and shows an overview of the measured mean circulation across the section. Typical crosssection velocities in the interior of the gyre amount to $0.5-1.0 \mathrm{~cm} / \mathrm{s}$ while at the continental slopes they are close to $4 \mathrm{~cm} / \mathrm{s}$ in the western outflow and $5-8 \mathrm{~cm} / \mathrm{s}$ in the eastern inflowing coastal jet. Along section velocity is less than $1 \mathrm{~cm} / \mathrm{s}$ in the interior and has typical values of $2-3 \mathrm{~cm} / \mathrm{s}$ near the shelves. These higher velocities are due to the fact that the section is not perpendicular to the small-scale depth contours. Standard deviations of the velocities are close to $1 \mathrm{~cm} / \mathrm{s}$ in general and increase to $2-5 \mathrm{~cm} / \mathrm{s}$ in the boundary currents.

The third type of data used in the analysis is the annual average wind stress curl over the Weddell Sea extracted from the data base of the European Centre for Medium Weather Forecasts (Glowienka-Hense et al., 1992). It has been computed by taking the curl of the surface wind stress averaged over the period from 1979 to 1987.

Step-like bathymetry (Figs. 2,4) of the model was derived from the original echo-sounding data obtained at the sections. Horizontal gradients $\nabla H$ were extracted from the ETOPO bathymetric dataset available at Alfred Wegener Institute (AWI). No filtering was done prior to computation of $\nabla H$ by central differences.

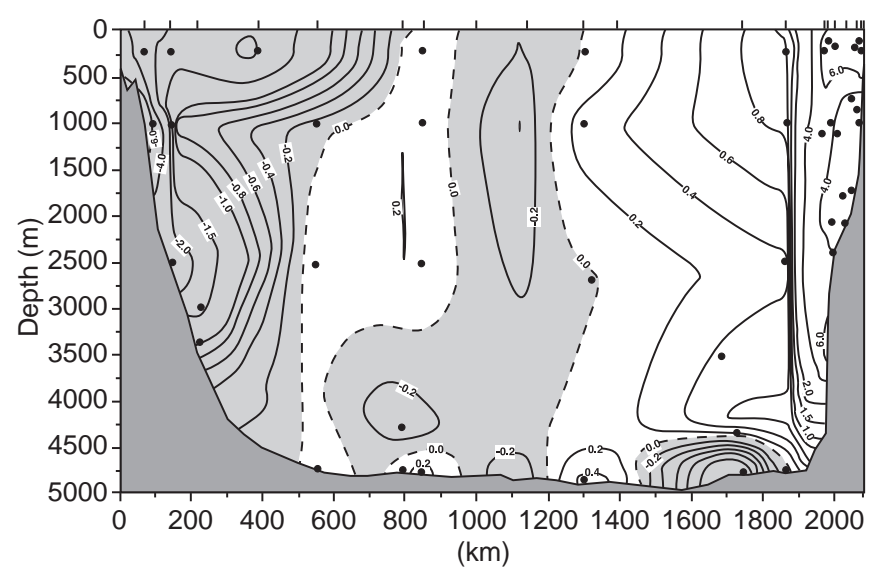

Fig. 3. Cross-section velocity interpolated by Fahrbach et al. (1994b) on the basis of the long-term moored measurements performed in the Weddell Sea. Contour interval is $0.2 \mathrm{~cm} / \mathrm{sec}$. Shaded areas denote outflows. Instrument locations are shown by solid circles

\section{The assimilation scheme}

\subsection{Setting of the problem}

Following the standard "assimilation philosophy", we consider all the data as a kind of spatio-temporal sample from a "random" ensemble of Weddell Gyre states. Treating the large-scale steady state of the Weddell Gyre as an average over this ensemble it is possible to find an approximation to this mean under the assumption that the prior probability distribution is Gaussian. Additionally a number of conservation laws and dynamical relationships must be satisfied (see Appendix). These relationships include geostrophy, hydrostatics and a non-linear equation of state for sea water. Furthermore the linearized potential vorticity balance and advective property balances are assumed to be in steady state. Finally an integral kinematic constraint imposes no net mass transport across the section. The last three of these constraints Eqs. (A3.2-A5) are imposed in a "weak" form, i.e. they are to be satisfied only within certain error bounds $F_{n}, F^{w}, F_{w}, F_{V}$ defined by a priori statistical assumptions. Geostrophy and the equation of state are satisfied exactly.

Our finite difference model is based on three-dimensional grid boxes in the section plane (Nechaev and Yaremchuk, 1995). Vertices of the boxes are defined by a regular mesh with homogeneous spacing of $35 \mathrm{~km}$ in the horizontal and uneven spacing in the vertical. There are 62 grid points along section line, 2 points across and 19 levels. The vertical section is defined by the measurements passes through the centers of the grid cells whereas hydrographic fields are estimated on the grid points, i.e. on both sides of the section plane. By this method the model allows us to calculate not only the mean values of the hydrographic fields (which should be close to the measurements) but also their cross-section gradients.

After the specification of the probability distribution and the dynamical constraints we assimilate the data into the model by seeking the most probable state of the Weddell Sea on our model grid $\Omega$ under the assumption that dynamical constraints outlined are satisfied. The numerical technique for that procedure is well established, (e.g. Le Dimet and Talagrand, 1986). The prior probability density function is proportional to $\exp \left(-J_{0}\right)$ where the argument $J_{0}$ of the Gaussian exponent has the form of the following quadratic cost function:

$$
\begin{aligned}
J_{0}= & \frac{1}{2}\left(\sum_{m, n}^{*}\left(\hat{I} C_{m}-C_{m}^{*}\right)^{\dagger} W^{m n}\left(\hat{I} C_{n}-C_{n}^{*}\right)\right. \\
& +\sum^{*}\left(\mathbf{u}-\mathbf{u}^{*}\right)^{\dagger} W_{u}\left(\mathbf{u}-\mathbf{u}^{*}\right)+W_{V} F_{V}^{2} \\
& +W_{w} \int_{z=-H}\left(F_{w}\right)^{2} \mathrm{~d} x+W^{w} \int_{z=0}\left(F^{w}\right)^{2} \mathrm{~d} x \\
& +\int\left(\sum _ { n } \left[F_{n}^{\dagger} W_{F}^{n} F_{n}+\left(\hat{S}_{1} C_{n}\right)^{\dagger} D^{n}\left(\hat{S}_{1} C_{n}\right)\right.\right. \\
& \left.\left.\left.+\left(\hat{S}_{1} F_{n}\right)^{\dagger} D_{F}^{n}\left(\hat{S}_{1} F_{n}\right)\right]+\left(\hat{S}_{2} \mathbf{u}\right)^{\dagger} D_{\mathbf{u}}\left(\hat{S}_{2} \mathbf{u}\right)\right) \mathrm{d} \Omega\right) .
\end{aligned}
$$


The first two terms in $J_{0}$ attract the solution to the data: $C_{m}^{*}$ and $C_{n}^{*}$ stand for the measured values of the $m$ th $(n \mathrm{th})$ property component and $\mathbf{u}^{*}$ identifies the long-term averages of the velocity data. $W^{m n}$ and $W_{u}$ are the inverse covariance matrices accounting for the prior statistical structure of the hydrographic properties $C_{n}$ and velocity $\mathbf{u} \equiv(u, v)$ fields respectively. $\hat{I}$ is a local interpolation operator which projects the unknown "steady state" field $C_{n}$ onto a measurement point. Asterisked summations are taken over the entire sets of hydrographic and velocity data points.

The remainder of the cost function is aimed at diminishing the "errors" in the determination of various components of the unknown steady state. Therefore we assume a priori that all the arguments in the last seven quadratic functions are statistically independent and have zero means. The different contributions describe: uncertainty of the net cross-section volume transport $F_{V}$, uncertainties in the vertical velocity at the bottom $F_{w}$ and on the sea surface $F^{w}$, deviations $F_{n}$ of the property conservation equation from the steady advective balance and finally variations of the grid-scale components of $C_{n}, F_{n}$ and $\mathbf{u}$. The latter are represented by applying differential operators $\hat{S}_{i} \equiv \partial_{x x}+v_{i}(z) \partial_{z z}$ to corresponding fields with weighting coefficients for the vertical $v_{i}$ depending on the type of field. Integrals are taken over the entire model grid $\Omega$ if not otherwise mentioned.

To find the maximum of the probability distribution we have to calculate the minimum of the cost function $J_{0}$. This optimization problem where the dynamical equations act as constraints is solved iteratively using the adjoint method. The iterative optimization procedure starts from some guess of the model state the first guess state. Due to non-linearity of dynamical constraints the cost function may have multiple minima and the result of optimization may depend on the choice of the first guess model state. Therefore, it is necessary to select the first guess as close to reality as possible.

Concerning the section data it is quite straightforward to obtain a reliable first guess for components of the model state corresponding to along section variability of oceanic fields. This is done by linear interpolation of the hydrographic data onto $\Omega$ followed by a dynamical computation of the cross-track velocity under the assumption of no motion at the bottom. But the information on cross-section gradients of the hydrographic fields and along-section velocities is not usually available. In the absence of this information the best guess for cross-track gradients and along-track velocity is obviously zero although this guess might be far from reality.

The procedure for improving the first guess for crosssection gradients and the dependence of the assimilation results on the first guess state are addressed in Sect 4.5. More details connected with application of the assimilation technique to section data sets can be found in Nechaev and Yaremchuk (1995).

\subsection{Prior variances}

Special attention should be drawn to the determination of the inverse variances $W^{m n}, W_{u}, W_{V}, W_{w}, W^{w}, W_{F}^{n}, D^{n}$, $D_{F}^{n}$ and $D_{u}$. These quantities define "physically acceptable" deviations of the state vector components from their steady state geostrophically balanced counterparts. In other words both $W$ and $D$ values should be inversely proportional to the squared amplitudes of the appropriate fields generated by ageostrophic motions and measurement errors. We assume that all these fields are statistically homogeneous in time and in the horizontal. Another assumption about these "sub-grid" fields is that they are uncorrelated in space and time.

Estimation of the $W$ and $D$ values is based on the variances of the available data and first guess model state. At the beginning, covariances of $C_{m n}=\operatorname{cov}\left(C_{n}, C_{m}\right)$ and variances $\sigma$ of $F_{n}, \hat{S}_{1} C_{n}, \hat{S}_{1} F_{n}, \hat{S}_{2} \mathbf{u}, F^{w}$ and $F_{w}$ are computed as standard deviations of these quantities from the means which have been taken over the domains of their statistical homogeneity, i.e. they are functions of depth, but do not depend on the location along the section. In order to estimate an "ageostrophic noise level" we computed the horizontal wave number spectra of all the properties at various depths. Fourier amplitudes corresponding to small-scale wavelengths were then associated with the "noise", which appeared to contribute approximately $30 \%$ to the net horizontal variance. Hence we roughly assume that our measurements sample the large-scale state with $30 \%$ error.

1. In accordance with these described preliminary analysis, we set $W^{m n}=10 C_{m n}^{-1}$. By doing this, we implicitly assume that in situ correlations between different properties at ageostrophic scales are the same as the ones derived from the data by standard statistical analysis in the horizontal.

2. The value of $\sigma(\mathbf{u})$ was found to be $1.3 \mathrm{~cm} / \mathrm{s}$. Following the mentioned estimate of the ageostrophic noise, we set $W_{u}=10 \sigma(\mathbf{u})^{-2}$. This choice corresponds to the rms error in sampling of the mean current by moorings of $0.4 \mathrm{~cm} / \mathrm{s}$.

3. Physically sensible considerations show that volume imbalance cannot be larger than a few tenths of a Sverdrup. Hence we set $W_{V}=80 \mathrm{~Sv}^{-2}$.

4. The rms error in the upper layer Ekman pumping rate derived from ECMWF data was found to be approximately $5 \mathrm{~m} / \mathrm{y}$, whereas the bottom Ekman layer pumping rate which is believed to be the major source of the uncertainty in the bottom boundary condition, was assumed to be of the order of $2 \mathrm{~m} / \mathrm{y}$.

5. $F_{n}$ may be interpreted as a source term for property $C_{n}$ which is necessary to keep the solution at steady state (see Appendix). First guess values of $F_{n}$ were on the order of magnitude of the major constituents in the 3D advective balance. It is physically sensible, however, to assume that advective balance should be satisfied to an accuracy of a few per cent, at least in the open ocean regions. This assumption is implicitly supported by known estimates of water mass transformation rates in the Weddell Sea, which show that 
typical values of the non-advective terms (which may also include residual temporal trends) south of the section are 10-20 times smaller than our first guess estimate. Therefore we set $W_{F}^{n}=100\left[\sigma\left(F_{n}\right)\right]^{-2}$.

6. Grid-scale components of the steady state fields $C_{n}$ and $F_{n}$ should be suppressed in a climatological mean. We do so by reducing the first guess estimates of their variances $20-30$ times, setting $D^{n}=50\left[\sigma\left(\hat{S}_{1} C_{n}\right)\right]^{-2}$ and $D_{F}^{n}=500\left[\sigma\left(\hat{S}_{1} F_{n}\right)\right]^{-2}$. On the other hand, high horizontal wave numbers should not be removed to the same extent from the velocity fields. This is due to the fact that velocities are dynamically connected to temperature and salinity by horizontal differentiation operators. Therefore we set $D_{u}=20\left[\sigma\left(\hat{S}_{2} \mathbf{u}\right)\right]^{-2}$ and introduce high anisotropy in $\hat{S}_{2}$ by taking $v_{2}=10 v_{1}$, so that the horizontal roughness of the velocity is penalized 10 times less than its vertical counterpart.

After the careful definition of $J_{0}$ a standard minimization routine was applied (Gilbert and Lemaréchal, 1989, routine M1QN3 of the MODULOPT library). The minimization performed a search in the space of control variables $\left\{C_{n}, \mathbf{u}(z=-H), F^{w}, F_{w}\right\}$ for the optimal state. Several thousand iterations were performed until convergence was achieved.

\subsection{Posterior variances}

The error analysis was the most expensive part of the present study from the computational point of view. Variances of all the integral quantities shown in Tables 2-5 were computed through linear transformations of the covariance matrix $K$ between the control variables. $K$ is identified as the inverse of the Hessian matrix $H$ associated with the assimilation scheme (Thacker, 1989).

Let us consider as an example the algorithm for computation of the variance $\sigma^{2}$ for the section mean value $\bar{F}_{n}$ of the source for the property $C_{n}$. First we compute the components of the vector $L$ which relates small fluctuations of control vector $X$ around optimal value with variation of $\bar{F}_{n}$. This can be done using the adjoint code:

$L=\frac{\delta \bar{F}_{n}}{\delta X}=\mathscr{D}_{o p t}^{\dagger} \frac{1}{\Omega} \frac{\delta}{\delta F_{n}} \int_{\Omega} F_{n} \mathrm{~d} \Omega$,

where $\mathscr{D}_{o p t}^{\dagger}$ is the adjoint of the dynamical operator of our model, linearized in the vicinity of the optimal state.

Following the standard approach (Thacker, 1989), we assume that posterior statistics are also Gaussian and fluctuations around the optimal state are small in the sense that original dynamics is well approximated by linearized equations. Then the variance can be calculated as

$\sigma^{2}\left(\bar{F}_{n}\right)=L^{\dagger} H^{-1} L$.

As the number of control variables was fairly large (17764-13348, depending on the type of data set) it is computationally prohibitive to calculate $H$ and especially $H^{-1}$. However it is possible (using the model and its adjoint) to calculate the product of the Hessian matrix $H$ and any vector $X$ (Le Dimet and Talagrand, 1986). A standard routine of the NAG library allows to find an iterative solution for large and sparse linear systems of the type $H X=L$, where only the right-handside must be given and subroutine has to be supplied that calculates the product $H X$ for any $X$. Transformation of $H X=L$ leads to $X=H^{-1} L$ and by insertion into Eq. (2) the variance can be calculated as $\sigma^{2}\left(\bar{F}_{n}\right)=L^{\dagger} X$.

Each variance calculation consumed 3 to $4 \mathrm{~h}$ of CPU on a Cray YMP/EL. As may be expected, practically all error estimates for the composite data set were found to be smaller than for any of the data sub-sets. Results are summarized in Tables $2-5$. They are also intensively used in the following discussion of the assimilation experiments.

\section{Results of assimilation}

\subsection{Mean fields}

Four major assimilation experiments were performed. Each section was analyzed separately and additionally the best estimate was calculated from the composite data set. In all four cases current meter data are included in the calculation. The resulting temperature fields, i.e. the average of the model temperature north and south of the section, are shown in Fig. 4a to 4d. They closely resemble the data (Fig. 2a to 2d) and have only slightly smoother gradients. Mean temperatures are -0.126 , $-0.112,-0.090$ and $-0.105^{\circ} \mathrm{C}$ respectively.

Table 1 gives an idea of the relative distances between the assimilated $(A)$, measured $(D)$ and the first guess $(F)$ temperature fields. These distances were computed through the following relationships:

$$
\begin{aligned}
& \mathscr{R}_{F D}=\frac{1}{\Omega} \int_{\Omega}\left[\left(\hat{I} T_{F}-T_{D}\right)^{2} / \sigma_{x}^{2}\left(T_{D}\right)\right] \mathrm{d} \Omega, \\
& \mathscr{R}_{A D}=\frac{1}{\Omega} \int_{\Omega}\left[\left(\hat{I} T_{A}-T_{D}\right)^{2} / \sigma_{x}^{2}\left(T_{D}\right)\right] \mathrm{d} \Omega, \\
& \mathscr{R}_{A F}=\frac{1}{\Omega} \int_{\Omega}\left[\left(T_{A}-T_{F}\right)^{2} / \sigma_{x}^{2}\left(T_{D}\right)\right] \mathrm{d} \Omega .
\end{aligned}
$$

Here $\sigma_{x}\left(T_{D}\right)$ denotes the depth-dependent horizontal mean square variation of the data.

Table 1 shows that the composite data set is characterized by higher misfits both for the first guess and assimilated fields. These misfits have the following origin: the composite data set has approximately 2.8 data points per grid cell, which may contradict each other, while other data sets have 1 data point per cell on the average, and a closer (but statistically less reliable) linear interpolation fit can be obtained. However, the magnitude of the misfit that remains after assimilation is relatively small and agrees with our a priori assumptions.

We may treat distances $\mathscr{R}_{A F}, \mathscr{R}_{A D}$ and $\mathscr{R}_{F D}$ as side lengths of corresponding triangles located in 

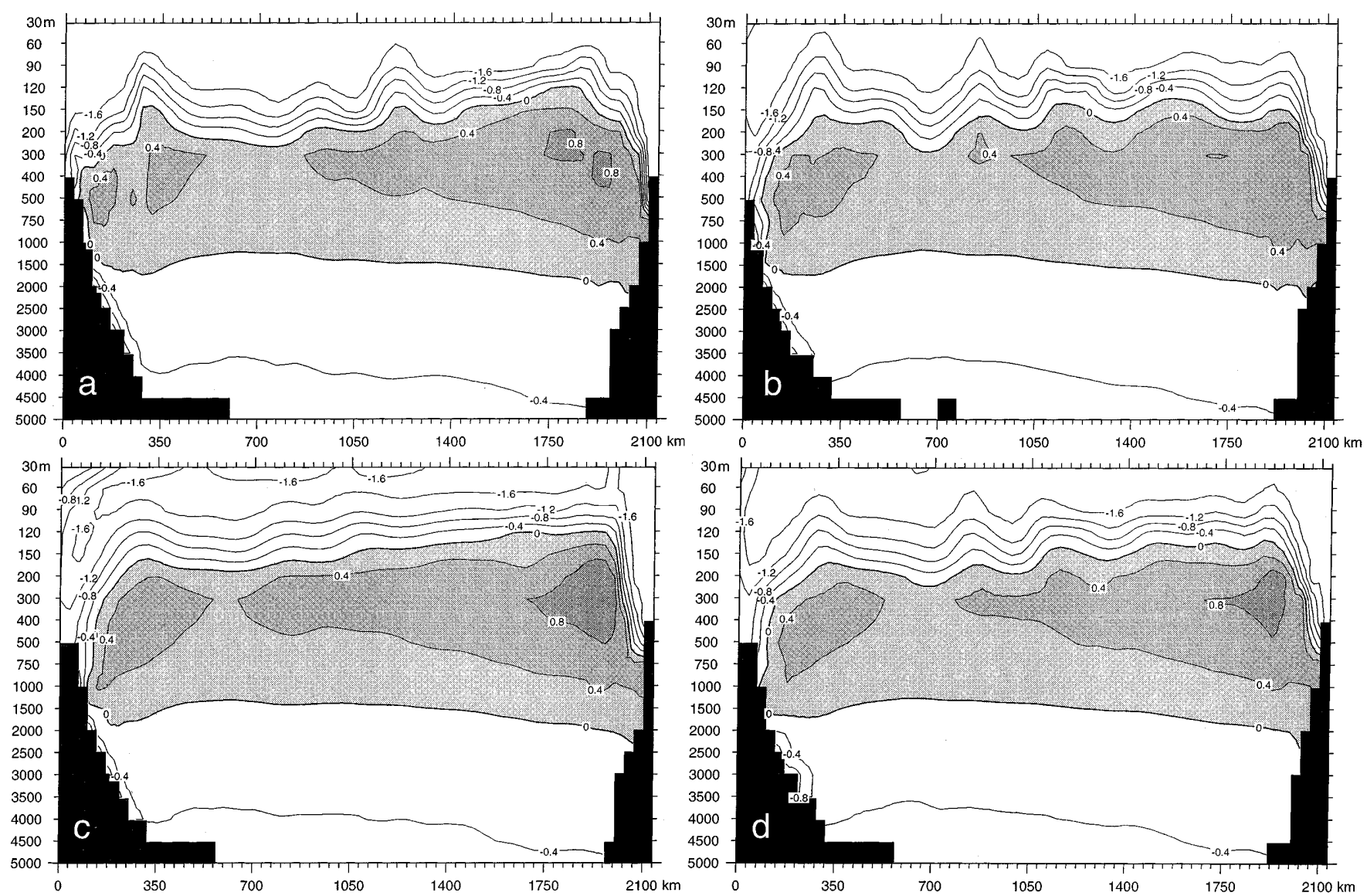

Fig. 4a-d. Temperature fields obtained by variational assimilation of the data measured in a 1989, b 1990 and c 1992-1993; d shows the result of assimilation of the composite data set

"temperature space". We note that angles between directions, pointing from data to first guesses and to optimal states are quite large and vary within the range of $87^{\circ}-119^{\circ}$ in all cases except the composite data set where it amounts to $39^{\circ}$. This indicates that assimilation provides us with states whose deviations from the data have rather small projections onto the ones derived by standard dynamical analysis.

Considering the residuals of the advective balance after assimilation, i.e. the distribution of $F_{n}$, we find mostly small-scale structures. An exception is found in the uppermost $200 \mathrm{~m}$ where the sources $F_{n}$ show a coherent pattern that resembles fluxes due to turbulent mixing. Section mean values of practically all property sources $\bar{F}_{n}$ shown in Table 2 cannot be confidently distinguished from zero, with two exceptions: the enrichment in $\mathrm{O}_{2}$ in 1990 and the temperature trend for the composite data set of $-0.0035^{\circ} \mathrm{C} / \mathrm{y}$.

Table 1. Distances between temperature fields

\begin{tabular}{llll}
\hline Data set & $\mathscr{R}_{A D}$ & $\mathscr{R}_{A F}$ & $\mathscr{R}_{F D}$ \\
\hline 1989 & 0.27 & 0.34 & 0.22 \\
1990 & 0.23 & 0.31 & 0.15 \\
1992 & 0.21 & 0.28 & 0.11 \\
$1989-1992$ & 0.38 & 0.24 & 0.32 \\
\hline
\end{tabular}

As a summary we may report that the assimilated temperature is in good agreement with the observations. The same holds for the other properties (not shown). A good representation of the hydrographic fields is the condition for a meaningful calculation of derived quantities such as transports and fluxes.

\subsection{Transports and fluxes}

Transports of various water properties through the section reflect the overall magnitude and direction of physical processes occurring in the Weddell Sea. After assimilation we can easily obtain values of transports $\Phi_{n}$ by integration over the section plane:

$\Phi\left(C_{n}\right)=\int_{\Omega} v C_{n} \mathrm{~d} \Omega$.

Results of these computations are assembled in Table 3. Variances of the transport estimates were computed through the covariance structure analysis Eq. (2). Values for most of the transport estimates for properties and for the water mass transformation rates are much larger than their standard deviations. These values may be considered as statistically reliable. It should be stressed that "reliability" in the sense of the computed error bars is entirely defined by the prior statistical 
Table 2. Tracer sources $\bar{F}_{n}$

\begin{tabular}{|c|c|c|c|c|c|c|c|c|}
\hline Data set & $\begin{array}{l}\mathrm{T} \\
10^{-4}{ }^{\circ} \mathrm{C} / \mathrm{y}\end{array}$ & $\begin{array}{l}\mathrm{S} \\
10^{-4} \mathrm{y}^{-1}\end{array}$ & $\begin{array}{l}\mathrm{O}_{2} \\
10^{-8} \mathrm{M} / 1 \mathrm{y}\end{array}$ & $\begin{array}{l}\text { Silicate } \\
10^{-8} \mathrm{M} / 1 \mathrm{y}\end{array}$ & $\begin{array}{l}\text { Nitrate } \\
10^{-9} \mathrm{M} / 1 \mathrm{y}\end{array}$ & $\begin{array}{l}\text { Phosphate } \\
10^{-10} \mathrm{M} / 1 \text { y }\end{array}$ & $\begin{array}{l}\text { Ammonium } \\
10^{-10} \mathrm{M} / 1 \mathrm{y}\end{array}$ & $\begin{array}{l}\mathrm{CO}_{2} \\
10^{-10} \mathrm{M} / 1 \mathrm{y}\end{array}$ \\
\hline 1989 & $-7 \pm 25$ & $-4 \pm 24$ & $11 \pm 31$ & $-7 \pm 19$ & $-8 \pm 26$ & $-5 \pm 41$ & $-6 \pm 17$ & - \\
\hline 1992 & $-10 \pm 22$ & $-17 \pm 23$ & $21 \pm 24$ & $-13 \pm 26$ & $-22 \pm 21$ & $-17 \pm 32$ & - & $-13 \pm 18$ \\
\hline 1989-1992 & $-35 \pm 19$ & $-5 \pm 21$ & $18 \pm 20$ & $-13 \pm 23$ & $-6 \pm 25$ & $-1 \pm 27$ & - & - \\
\hline
\end{tabular}

Table 3. Transport estimates

\begin{tabular}{|c|c|c|c|c|c|c|c|c|c|}
\hline Data set & $\begin{array}{l}\mathrm{V} \\
\mathrm{Sv}\end{array}$ & $\begin{array}{l}\mathrm{T} \\
10^{12} \mathrm{~W}\end{array}$ & $\begin{array}{l}\mathrm{S} \\
10^{4} \mathrm{~kg} / \mathrm{s}\end{array}$ & $\begin{array}{l}\mathrm{O}_{2} \\
10^{3} \mathrm{M} / \mathrm{s}\end{array}$ & $\begin{array}{l}\text { Silicate } \\
10^{3} \mathrm{M} / \mathrm{s}\end{array}$ & $\begin{array}{l}\text { Nitrate } \\
10^{2} \mathrm{M} / \mathrm{s}\end{array}$ & $\begin{array}{l}\text { Phosphate } \\
10 \mathrm{M} / \mathrm{s}\end{array}$ & $\begin{array}{l}\text { Ammonium } \\
10 \mathrm{M} / \mathrm{s}\end{array}$ & $\begin{array}{l}\mathrm{CO}_{2} \\
10 \mathrm{M} / \mathrm{s}\end{array}$ \\
\hline 1989 & $32 \pm 4$ & $-35 \pm 12$ & $51 \pm 35$ & $24 \pm 58$ & $138 \pm 79$ & $35 \pm 62$ & $36 \pm 58$ & $62 \pm 48$ & - \\
\hline 1992 & $35 \pm 4$ & $-22 \pm 10$ & $73 \pm 38$ & $84 \pm 52$ & $75 \pm 68$ & $77 \pm 67$ & $160 \pm 52$ & - & $74 \pm 87$ \\
\hline 1989-1992 & $34 \pm 2$ & $-28 \pm 8$ & $72 \pm 26$ & $45 \pm 49$ & $95 \pm 66$ & $21 \pm 58$ & $180 \pm 47$ & - & - \\
\hline $1989-1990^{\mathrm{a}}$ & $29 \pm 9$ & $-35 \pm 10$ & $10 \pm 22$ & - & - & - & - & - & - \\
\hline
\end{tabular}

${ }^{a}$ Estimates made by Fahrbach et al. (1994)

assumptions outlined by the cost function. An idea about the reliability of these hypothesis can be obtained from the variability of the estimates from one data set to another.

The major characteristic of the cross-section circulation is the net transport of the Weddell Gyre which was computed by integrating $v$ over the domain west of $17^{\circ} \mathrm{W}$ (left of the $1750 \mathrm{~km}$ marker in Fig. 4). The values obtained for different data sets (Table 3 ) range between 32 and $35 \mathrm{~Sv}$ and are in agreement with the estimate of Fahrbach et al. (1994), who obtained $29.5 \pm 9.5$ Sv. Our estimates have a posterior rms discrepancy with the velocity measurements of 0.5 to $1.2 \mathrm{~cm} / \mathrm{s}$. The transport value of $34 \mathrm{~Sv}$ from the composite data set was used in a different assimilation study to constrain a three dimensional inverse model of the Weddell Sea (Nechaev et al., 1995).

The heat transport for different data sets ranges within $22-35 \times 10^{12} \mathrm{~W}$ (column 2, Table 3 ). This relatively small range demonstrates the high stability between different data sets. Dividing this value by the sea surface area south of the section results in $Q^{*}=12-18 \mathrm{Wm}^{-2}$ as an estimate for the mean cooling rate in the Weddell Sea. This value agrees rather well with the $16 \mathrm{Wm}^{-2}$ derived by Gordon and Huber (1990).

Salt transport results in a statistically significant salt outflow $\Phi(S)$ of between 500 to 700 tons/s for different data sets. However, there should be no net transport of salt in a steady state balance. We may attribute this discrepancy to sea ice which is not included in our model. Both volume and salt can be conserved if we allow a corresponding ice export. Assuming an average salinity for sea ice of $5 \mathrm{ppt}$ we interpret the salt flux as a net ice export of $530 \mathrm{~km}^{3} / \mathrm{y}$.

While Weddell Gyre volume and heat transports are in good agreement with the estimate Fahrbach et al.
(1994), our net salt flux is not. Fahrbach et al. (1994) obtained no net salt flux which indicates a compensation of the salt release by ice formation through fresh water input. We explain this discrepancy in two ways: the differences in the upper ocean current field and the used hydrographic data. In the previous estimate the velocities are constant above the uppermost current meter while in this study they vary vertically according to the dynamical constraints. In the upper ocean we also measure vertical gradients of salinity whereas, in the remainder of the water column, salt content varies only a little. Due to high spatial variability of the upper ocean current and salinity the obtained salt transport is very sensitive to changes in the used data. Therefore the step from a pure summer hydrographic data set used by Fahrbach et al. (1994) to the averaged data used here has a significant effect on the results.

Transports of passive properties are calculated in Mol per second and also shown in Table 3. Although their variances are rather large they support the view that water in the southwestern Weddell Sea is enriched by oxygen and nutrients. The latter may originate from the remineralization processes south of the transect. However, this requires oxygen consumption which is not consistent with the observed oxygen enrichment. We conclude that physical solution of oxygen in the surface layers and subsequent water mass formation leads to compensation of the oxygen loss due to remineralization.

In general, results shown in Table 3 support the view that transports of the steady state are more or less stable from year to year at least within the error bars. On the other hand, climatologically significant trends may also exist in reality, but since they are not adequately resolved by our data set, we could not take advantage of using time-dependent dynamical constraints. Looking at Table 3, one may have a rough idea of the magnitude of these trends. 


\subsection{Estimates of mixing rates}

Estimates for the transformation rates of water masses can be obtained in two ways. The first one is based on the analysis of the differences between sea water properties of the inward and outward flows. Alternative estimates can be derived from the fields of property sources $F_{n}$ if we assume that their physical background is mainly defined by the appropriate processes of smaller scales (mixing, deep convective adjustment, etc.).

Let us consider the first approach. Following Fahrbach et al. (1994), we divide the section area into four subdomains occupied by different water masses: surface water (SW), warm deep water (WDW), Weddell Sea deep water (WSDW) and Weddell Sea bottom water (WSBW). These water masses are identified by their characteristics in $\theta-S$-space (see Fig. 5). Integrating the cross-section velocity over these subdomains, we obtain an idea of the net water mass transformation rate in the Weddell Sea basin south of the transect. Results of these computations are assembled in Table 4. The last four columns in Table 4 demonstrate the relative contributions of these domains to the area of the section plane. The composite data indicate that inflow of the WDW splits into outflows of WSDW and WSBW which are almost equal. The contribution of the SW in this diagram appears to be statistically uncertain. This is in contrast with (Fahrbach et al., 1994), who find a significant transformation to SW. However, the discrepancy can be explained as well by the different near surface currents used in the two calculations.

The alternative estimate directly employs information extracted from the data by the assimilation scheme. The advective property balances are solved in a least squares sense. Small discrepancies remain after the assimilation. They have the dimension of time derivatives, which we interpret as intensity of non-advective processes. To study these trends they were integrated for one year and plotted as arrows in a $\theta$-S-diagram (Fig. 5a). Each arrow starts at assimilated $\theta-S$ values and points at the respective values one year later. It is remarkable how much the residual trends resemble mixing of water masses and describe a process that is not modeled explicitly. Actually, it is well known, e.g. Fahrbach et al. (1994), that the region is characterized by transformation of WDW into SW, WSDW and WSBW. The transformation WDW $\rightarrow \mathrm{SW}$ is the most visible one in Fig. 5a. Transition WDW $\rightarrow$ WSDW is not so evident

Table 4. Water mass transformation rates south of the section and areas of section plane occupied by water masses

\begin{tabular}{lllllllll}
\hline Data set & $\begin{array}{l}\text { WDW } \\
\text { Sv }\end{array}$ & $\begin{array}{l}\text { WSDW } \\
\text { Sv }\end{array}$ & $\begin{array}{l}\text { WSBW } \\
\text { Sv }\end{array}$ & $\begin{array}{l}\text { SW } \\
\text { Sv }\end{array}$ & $\begin{array}{l}\text { WDW } \\
\%\end{array}$ & $\begin{array}{l}\text { WSDW } \\
\%\end{array}$ & $\begin{array}{l}\text { WSBW } \\
\%\end{array}$ \\
\hline 1989 & $-4.6 \pm 1.5$ & $2.2 \pm 1.7$ & $2.1 \pm 2.0$ & $0.3 \pm 2.4$ & 23.2 & 65.1 & 6.5 \\
1990 & $-5.1 \pm 1.8$ & $0.9 \pm 2.0$ & $2.8 \pm 2.2$ & $1.2 \pm 2.7$ & 23.3 & 63.2 & 8.5 & 5.2 \\
1992 & $-3.9 \pm 1.5$ & $1.5 \pm 1.6$ & $2.2 \pm 2.1$ & $0.1 \pm 2.5$ & 23.7 & 65.0 & 6.4 & 5.0 \\
$1989-1992$ & $-6.6 \pm 1.1$ & $2.6 \pm 1.3$ & $2.5 \pm 1.9$ & $1.5 \pm 2.0$ & 24.3 & 63.1 & 7.5 & 5.1 \\
$1990^{a}$ & -6.0 & 1.2 & 2.6 & 2.2 & 25.6 & 63.3 & 6.1 & 4.9 \\
\hline
\end{tabular}

${ }^{a}$ Estimates made by Fahrbach et al. (1994)
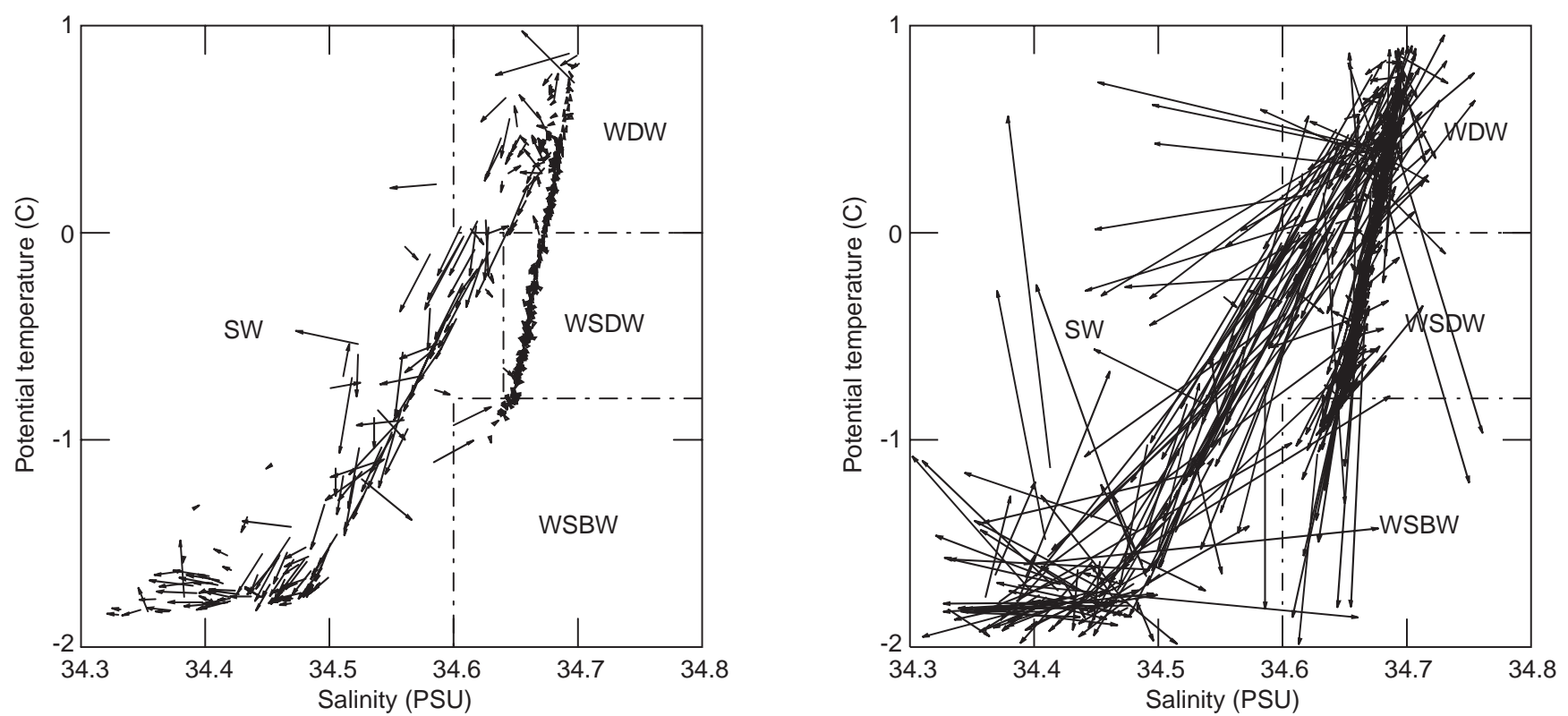

Fig. 5a,b. "Vector" $\theta-S$ diagrams derived a from the assimilation solution and $\mathbf{b}$ the first guess model state of the composite data set. Arrows denote annual mean displacements of the water parcels in the absence of advection. Parcels, leaving the plot area in $\mathbf{b}$ are not shown 
due to a relatively low magnitude and large volume where mixing processes of this kind occur. WSBW is formed indirectly from WDW through SW at the shelves. This fact is reflected by a weak "backward loop" in the lower part of the diagram.

In Fig. $5 b$ the same diagram is shown for the first guess model state. More than half of the arrows are not shown, because they end far beyond the margins of the figure. It can be seen that this state being only statistically consistent, exhibits strong temporal trends and a lot of scatter. By achieving a dynamically consistent solution the scatter is reduced considerably.

Transformation rates can be estimated from average travel times between pairs of water masses in the diagram shown in Fig. 5. In this respect "averaging" implies integration over all possible trajectories in the $\theta$ $S$-space, while the data available do not provide a sufficient statistics for such a procedure.

We can estimate, however, the overall effect of water mass transformation processes occuring in the domain, enveloped by our computational grid. This can be done by integrating of the source terms $F_{n}$ over $\Omega$ (Table 2 ). Local surface cooling rate can then be estimated by dividing figures in the first column by the area of the upper interface of $\Omega$. As a result we get $\dot{Q}=2 \pm 1 \mathrm{Wm}^{-2}$ for the composite data set. Of course, this estimate is valid under the assumption which neglects diffusive heat fluxes at the northern and southern boundaries of $\Omega$. Nevertheless, this figure corresponds to the results of direct measurements of the turbulent heat flux into the mixed layer under heavy ice conditions reported in Gordon et al.(1981). The discrepancy between the estimated local fluxes and the ones for the areas south of the transect of 12 to $18 \mathrm{Wm}^{-2}$, can be explained by the small contribution of shelf areas to the transect where the polynyas allow for an intense ocean-atmosphere exchange. This points to the significant contribution of the shelf areas to the water mass formation in the Weddell Sea.

Proceeding further on to the results of assimilation, we shall now consider data subsets which may give more insight into the properties of the employed assimilation algorithm.

\subsection{The role of velocity data}

As a rule, long-term velocity measurements in the open ocean are more expensive and provide larger uncertainties of the corresponding large-scale fields than CTD soundings and bottle samplings. Therefore the question to what extent do velocity data contribute to large-scale circulation pattern is still one of the major issues in oceanography.

Formally, the geostrophical steady state component of the oceanic circulation is governed by a set of equations which can locally identify the $3-\mathrm{D}$ velocity field given the proper distribution of active properties and their sources and sinks. Explicit expressions for velocity components (Needler, 1985) show, however, that the latter are non-linear functions of high-order derivatives of the "steady state" density field. Assuming that "steady state" signal/noise ratio for a CTD density profile in our region is equal to 3 (Sect. 3.2), and taking into account the mentioned dynamics (also used in our assimilation scheme), one can estimate that velocity signal/noise ratio must be 3-5 orders of magnitude lower. Therefore, if the large-scale hydrographic fields do contain information locally about the absolute velocity, then this information is confined to small-scale components of hydrographic fields (i.e. their high-order spatial derivatives).

Concerning the hydrographic section data, the valuable information on the large-scale components of crosssection velocity can be provided by the assumption of integral conservation of density within layers confined between isopicnal surfaces. For example, the wellknown method of the absolute velocity calculation on a closed hydrographic section (Wunsch, 1978) is based on this assumption. The layers usually extend over the entire domain separated by the section. The assumption can be justified if such layers (1) do not outcrop and (2) the integral mixing rates through isopicnal interfaces are negligible (or known). Neither of these conditions is satisfied in the Weddell Sea.

In the present study we utilize one integral constraint of total mass conservation (see Appendix, Eq. A5) and local dynamical constraints which have much in common with standard steady state circulation equations, e.g. Wunsch (1994). To reveal the influence of velocity data on the resulting state we conduct a number of experiments. In the first series of experiments we have assimilated all the available data using properties separately for the 1989, 1990 and 1992 sections and for the composite hydrographic data set. In the second series of experiments we have processed the same four data sets except for the velocity data, which were not taken into account by setting $W_{u}=0$.

Assimilated property fields from this second series of experiments are hardly distinguishable from the first series. However, the velocity fields and consequently the transports have changed considerably. Figure 6a displays the cross-section velocity pattern after taking the velocity data into account. For comparison our estimate without velocity measurements is shown in Fig. 6b. The major features of the current field remain qualitatively unchanged, but estimated velocities (especially near the continental slopes) decrease approximately by a factor of 0.5 in the case when velocity data are neglected. As a result, important integral parameters of the circulation are generally underestimated and statistically indistinguishable from zero (Table 5). Thus the Weddell Gyre transport increases from 9-17 Sv to 32-35 Sv between the series of experiments (compare Tables 3 and 5). The net cross-section heat flux varies within the ranges of $9 \times 10^{12}-1.7 \times 10^{13}$ and $2.2 \times 10^{13}$ $3.5 \times 10^{13} \mathrm{~W}$, respectively. The same is valid for net flux values of the other properties. On the other hand the presence of velocity data does not strongly affect property patterns. All changes are hardly distinguished by eye and contribute less than $1 \%$ to the properties' horizontal variances at any level. This fact can be 

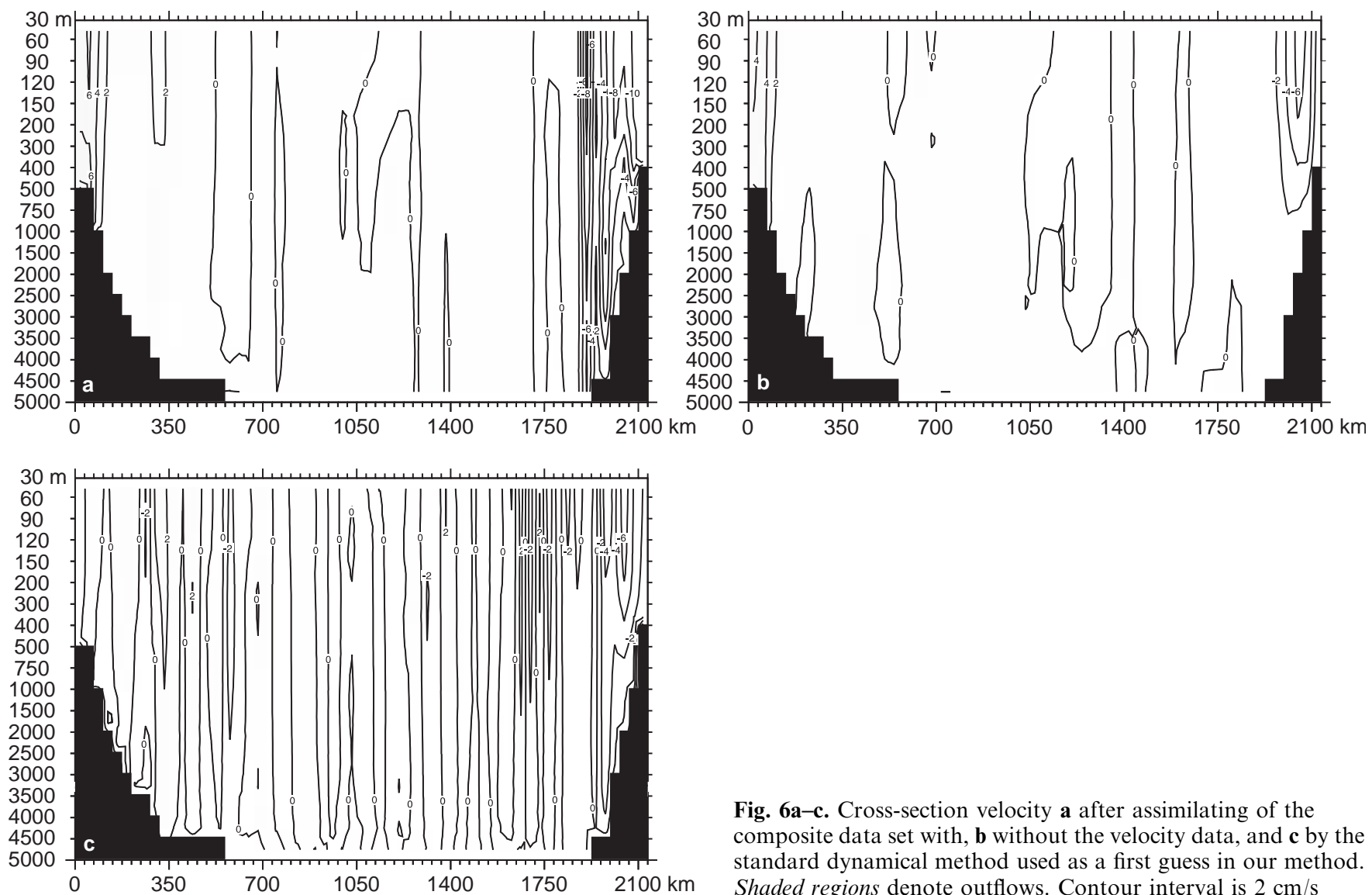

Fig. 6a-c. Cross-section velocity a after assimilating of the composite data set with, $\mathbf{b}$ without the velocity data, and $\mathbf{c}$ by the standard dynamical method used as a first guess in our method. Shaded regions denote outflows. Contour interval is $2 \mathrm{~cm} / \mathrm{s}$

explained by the mentioned properties of the dynamical constraints which are used in the assimilation algorithm.

Transport estimates for the assimilation without velocity data are shown in Table 5. Compared to results of Table 3, which included current meter data we notice much smaller transports and much higher error bars. Most of the transports cannot be regarded as significantly different from zero. Additionally the transport estimates for the first guess are shown in Table 6. As these are calculated under the assumption of zero velocity at the bottom corrected by the condition of zero cross-section transport, they may be considered as standard dynamical estimates. It is not meaningful to calculate error bars for the standard estimates, because our error analysis requires a converged solution. From Tables 5 and 6 it is clear that (1) our technique is superior to the standard analysis and (2) that current meter data have a highly beneficial impact on transport estimates and error reduction.

More drastic changes occur in error bars of integral quantities, which were computed on the basis of the probability distribution Eq. (1). As an example, typical value of the net volume transport variance decreases approximately four times as we take the velocity data into account. Another important feature is a seeming contradiction between volume transport estimates shown in Tables 3 and 5: only the 1990 transport estimate from Table 5 (without velocity data) seems to be consistent with the one obtained after taking this data into account. This is interpreted as a result of large impact given by velocity data to our prior probability distribution, which exhibits completely different behavior in the part that describes the velocities. One can expect that in ocean regions with stronger barotropic currents such impact can be even larger.

Table 5. Tracer transports without velocity data

\begin{tabular}{|c|c|c|c|c|c|c|c|c|c|}
\hline Data set & $\begin{array}{l}\mathrm{V} \\
\mathrm{SV}\end{array}$ & $\begin{array}{l}\mathrm{T} \\
10^{12} \mathrm{~W}\end{array}$ & $\begin{array}{l}\mathrm{S} \\
10^{4} \mathrm{~kg} / \mathrm{s}\end{array}$ & $\begin{array}{l}\mathrm{O}_{2} \\
10^{3} \mathrm{M} / \mathrm{s}\end{array}$ & $\begin{array}{l}\text { Silicate } \\
10^{3} \mathrm{M} / \mathrm{s}\end{array}$ & $\begin{array}{l}\text { Nitrate } \\
10^{2} \mathrm{M} / \mathrm{s}\end{array}$ & $\begin{array}{l}\text { Phosphate } \\
10 \mathrm{M} / \mathrm{s}\end{array}$ & $\begin{array}{l}\text { Ammonium } \\
10 \mathrm{M} / \mathrm{s}\end{array}$ & $\begin{array}{l}\mathrm{CO}_{2} \\
10 \mathrm{M} / \mathrm{s}\end{array}$ \\
\hline 1989 & $9 \pm 16$ & $-14 \pm 56$ & $22 \pm 156$ & $-24 \pm 209$ & $138 \pm 292$ & $-44 \pm 249$ & $14 \pm 194$ & $30 \pm 175$ & - \\
\hline 1992 & $12 \pm 14$ & $-11 \pm 49$ & $28 \pm 125$ & $99 \pm 202$ & $-26 \pm 283$ & $-21 \pm 216$ & $23 \pm 174$ & - & $5 \pm 371$ \\
\hline 1989-1992 & $13 \pm 6.5$ & $-9 \pm 37$ & $35 \pm 118$ & $-15 \pm 132$ & $35 \pm 227$ & $-1 \pm 178$ & $43 \pm 167$ & - & - \\
\hline
\end{tabular}


Table 6. Tracer transports by standard dynamical analysis

\begin{tabular}{|c|c|c|c|c|c|c|c|c|c|}
\hline Data set & $\begin{array}{l}\text { V } \\
\text { Sv }\end{array}$ & $\begin{array}{l}\mathrm{T} \\
10^{12} \mathrm{~W}\end{array}$ & $\begin{array}{l}\mathrm{S} \\
10^{4} \mathrm{~kg} / \mathrm{s}\end{array}$ & $\begin{array}{l}\mathrm{O}_{2} \\
10^{3} \mathrm{M} / \mathrm{s}\end{array}$ & $\begin{array}{l}\text { Silicate } \\
10^{3} \mathrm{M} / \mathrm{s}\end{array}$ & $\begin{array}{l}\text { Nitrate } \\
10^{2} \mathrm{M} / \mathrm{s}\end{array}$ & $\begin{array}{l}\text { Phosphate } \\
10 \mathrm{M} / \mathrm{s}\end{array}$ & $\begin{array}{l}\text { Ammonium } \\
10 \mathrm{M} / \mathrm{s}\end{array}$ & $\begin{array}{l}\mathrm{CO}_{2} \\
10 \mathrm{M} / \mathrm{s}\end{array}$ \\
\hline 1989 & 4.5 & 6.4 & 102 & -102 & 181 & 65 & 71 & 19 & - \\
\hline 1992 & 3.7 & -0.1 & 60 & -32 & 71 & 43 & 34 & - & 55 \\
\hline 1989-1992 & 8.0 & -4.2 & 379 & 28 & 144 & 134 & 86 & - & - \\
\hline
\end{tabular}

It is also worthwhile to mention that, even in the absence of velocity data, the variational assimilation provides us with better estimates of transports and fluxes, than the standard dynamical method. Estimates of bottom velocities are more reasonable.

A certain amount of information on these quantities may arrive from the data of bottom topography, wind stress and hydrographic field structure near the shelves. For comparison, the velocity field of the standard dynamical method (i.e. our first guess velocity field) is shown in Fig. 6c. Its structure is quite different from the ones depicted in Fig. 6a-b: high horizontal intermittency is induced by noise in the composite data set (see Fig. 2d) and appears to be rather unrealistic. Moreover the net cross-section volume transport amounts to -15 Sv, which also contradicts simple physical considerations. The net transport can be corrected by subtracting the value of $-15 \mathrm{~Sv} / 9200 \mathrm{~km}^{2}=-0.16 \mathrm{~cm} / \mathrm{s}$ from the velocity field, but the overall cross-section circulation pattern will remain qualitatively unchanged. Further ad hoc improvements of dynamical method results can be certainly done by horizontal smoothing of the velocity field and/or preprocessing data on temperature and salinity. However these will need a lot of oceanographic experience and implicit use of information derived from additional sources. Major advantage of the assimilation scheme is that the use of such additional information is formalized (at least within the limited framework of dynamical and statistical hypothesis outlined).

\subsection{Cross-section gradients}

A novel opportunity offered by our assimilation scheme is the possibility to obtain estimates of the properties' cross-section gradients together with the along section geostrophic velocity. Standard section data usually give fair coverage of the variability in $(x, z)$ plane, whereas cross-section direction $(y)$ is often not covered at all. Utilization of geostrophic and steady advective balance constraints allows us to redistribute information between all the directions in 3-D space. A certain contribution to this is made by along-section velocity data, if available.

In practice, however, the flow of information into the $y$-direction may encounter some technical obstacles. The major one is connected with the non-linearity of the assimilation problem. In general, the cost function Eq. (1) may have multiple minima in the space of its control variables and the quality of assimilation will depend on the quality of the first guess. Therefore it is necessary to select the first guess as close to reality as possible. In the absence of any information on crosssection variability the best guess for cross-track gradients and along-track velocity is obviously zero. Remnants of such a high anisotropy of the first guess state are often observed in interpolated fields, especially in cases when the velocity data are spatially sparse (see Fig. 3). Generally they manifest themselves in an underestimation of the cross-section gradients compared to typical values of their along-section analogues. To avoid this shortcoming we minimized the cost function in two separate steps. First we introduced our prior assumption that along-section gradients and crosssection gradients should be distributed isotropically in the horizontal by changing the metric of the space of control variables. This change acts as a kind of preconditioning in the iterative minimization of $J_{0}$ and it prefers model states with high cross-section gradients. When after a number of iterations an equilibrium in spatial variability is reached, the metric is changed back to an orthogonal mode, which distributes information between the directions more or less homogeneously. Taking the model state achieved so far as an improved first guess with non-zero cross-section gradients the minimization is continued. As a result we reach an optimal model state with isotropic horizontal variability in the core of the gyre where the velocity data are especially sparse.

The 1990 hydrographic data set provides a possibility to check the quality of assimilation result in the crosssection direction (see the triangular station distribution in Fig. 1). Originally this station series was performed to monitor the mesoscale feature seen in Fig. $2 b$ in the middle of the section. In order to extract large-scale property gradients we approximated their measured horizontal distributions (having 16 points on the average) by a plane at each level. Figure 7 shows a scatter plot of the measured large-scale temperature gradients versus their counterparts taken from the solution of the 1990 section data. To make the small gradients at depth visible each gradient value is normalized by the value of $\left(T_{i}^{\max }-T_{i}^{\min }\right) / \mathrm{d} x$ where $T_{i}^{\max }$ and $T_{i}^{\min }$ are the maximum and minimum values of $T$ at a given level $i$ and $\mathrm{d} x=35$ $\mathrm{km}$ is the horizontal step of the model grid. Though the statistically significant positive correlation is obvious, cross-section gradients (diamonds in Fig. 7) demonstrate much larger scatter than along-section gradients (crosses) since the model estimates are only indirectly calculated from along-track data. Another reason for this may be attributed to severe contamination of the 


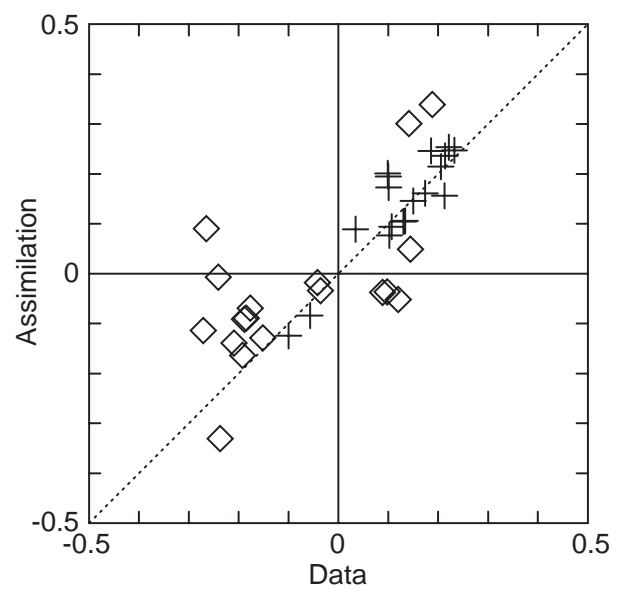

Fig. 7. Scatter plot of the along-section (crosses) and cross-section (diamonds) gradients of temperature at different levels obtained from the data taken on the triangular shaped track in 1990. "Assimilation" values are taken from the solution pattern. "Data" values are obtained by the direct linear fit of the data

large-scale signal by the mesoscale disturbance seen in Fig. 2b. On the average, the mean (over all the eight properties) correlation coefficient between the alongsection gradients was found to be 0.58 . Cross-section gradients are correlated by only 0.34 . Temperature and salinity demonstrate 0.49 correlation in $y$-variability. Passive properties have an average correlation coefficient of 0.29 . What is perhaps more important than correlation coefficients are the regression coefficients which are close to one in all cases.

Along-section geostrophic velocity depicted in Fig. 8 shows reasonable behavior at least in the core of the gyre with dominating northwestward transport which agrees both with sign of the gyre rotation and with the local bottom topography pattern.

From our point of view these facts give an additional support to the validity of the assimilation scheme.

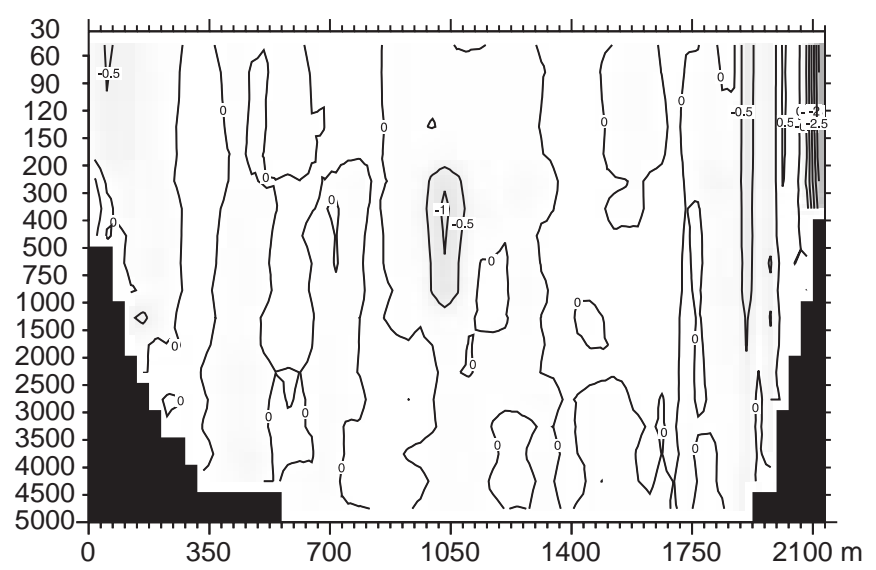

Fig. 8. An "optimal" estimate of the along-section geostrophic velocity component of the mean Weddell Gyre state. Regions of westward currents are shaded. Contour interval is $0.5 \mathrm{~cm} / \mathrm{s}$

\section{Summary}

The least squares dynamically constrained optimization technique has a number of advantages over more standard methods which often use dynamical and statistical principles separately. The utilized approach enables one to combine an arbitrary set of measured quantities, such as bottle samplings, long-term mooring data and atmospheric climatology.

The study presented is substantially based on the assumption of existence of a steady state circulation which satisfies standard large-scale dynamical constraints within the error bars outlined in Sect. 3.2. Such an assumption is meaningful if the state vector components (3-D velocity vector and property fields) do not vary much during the observation period. Rigorous error analysis of the optimal states have shown that our assumption is realistic, at least in the sense of integral transports, which were found to vary in time within the range of standard deviations of their error estimates (Table 3). Nevertheless, existence of relatively strong trends can not be ruled out, but they are hardly detectable in assimilation results by purely statistical means. More accurate determination of these trends requires incorporating time dependent dynamical constraints in conjunction with statistics. Unfortunately, temporal resolution of the hydrography is far too low for application of a time-dependent assimilation scheme.

Major results of the assimilation can be viewed as follows. In the period between September 1989 and January 1993 the Weddell Sea state was characterized by:

1. The Weddell Gyre transported water at the average rate of $34 \pm 2 \mathrm{~Sv}$. By the use of dynamical constraints uncertainty of the transports could be reduced significantly.

2. Heat was transported poleward at the rate of $28 \pm 8 \times 10^{12} \mathrm{~W}$ which translates into $15 \pm 3 \mathrm{Wm}^{-2}$ as an estimate of the mean cooling rate of the Weddell Sea south of the transect. This exceeds the estimated local flux on the transect which is obtained as $2 \pm 1 \mathrm{Wm}^{-2}$. As the transect extends mostly into the open ocean, we conclude that the shelf areas contribute significantly to the ocean-atmosphere exchange and are consequently the key areas for the contribution of the Weddell Sea to global ocean ventilation.

3. Salt transport estimates give an indication of a net ice outflow from the Weddell Sea of $530 \pm 250 \mathrm{~km}^{3} / \mathrm{y}$ $\approx 0.017 \mathrm{~Sv}$.

4. The derived salt transport is highly sensitive to the upper ocean current pattern and interseasonal variability.

5. Passive properties (oxygen, silicate, nitrate, phosphate, ammonium and carbon dioxide) were transported out of the Weddell Sea at rates of $45 \pm 49 \times 10^{3}, 95 \pm 66 \times 10^{3}, 21 \pm 58 \times 10^{2}, 1800 \pm$ $470,470 \pm 510$ and $740 \pm 870 \mathrm{M} / \mathrm{s}$ respectively. In particular, these figures give an indication that surface ventilation compensates oxygen consumption by remineralization. 
6. Processes occurring south of the section transformed $6.6 \pm 1.1 \mathrm{~Sv}$ of the inflowing warm deep water into approximately equal amounts of Weddell Sea deep water and Weddell Sea bottom water. Newly formed surface water was completely incorporated in the newly formed deep and bottom water, so that the volume transport of the in- and outflow did not differ significantly.

7. It has been shown that current meter data had a major impact on the solution. Only by taking them into account we arrived at reasonable and reliable estimates of the different transports and their variability.

8. The differences between the present results and those of Fahrbach et al. (1994) underline the sensitivity of the estimates to the quality of the description of the upper ocean conditions. This holds in particular for the coastal areas because the major ocean-atmosphere exchange occurs at the shelf as indicated by the calculated heat flux.

As for any other assimilation algorithm, these results may contain imprints of our subjectiveness in formulation and interpretation of the method. Nevertheless we hope that the estimates presented may give a valuable contribution to quantitative understanding of the physical processes taking place in the Weddell Sea.

The type of analysis described here can be used for other WOCE sections. In our solution the current measurements constrained the solution quite strongly. For other areas we may therefore suggest that either current meter velocities or other independent data like satellite altimetry should be used in order to estimate transports that are statistically reliable.

\section{Appendix}

All the assimilated fields satisfy the following constraints:

1. Geostrophic relationships:

$\mathbf{u}-\frac{g}{\rho_{0} f} \int_{-H}^{z}(\mathbf{k} \times \nabla \rho) \mathrm{d} z-\mathbf{u}_{b}=0$,

where $\mathbf{u}_{b}$ is the velocity at the bottom $z=-H(x, y)$, the rest of notation is standard.

2. The equation of state of sea water (Fofonoff and Millard, 1983):

$\rho-R(T, S)=0$.

3 . The relationship for the vertical velocity $w$ derived by taking the vertical derivative of the linearized steady state equation for conservation of quasi-geostrophic potential vorticity is:

$\frac{\partial^{2} w}{\partial z^{2}}-\frac{g}{\rho_{0} f^{2}}(\nabla \rho \times \mathbf{k}) \cdot \nabla f=0$

with boundary conditions:

$w(0)-\left(\frac{1}{f} \operatorname{curl} \tau+F^{w}\right)=0$,
$w(-H)-\left[\left(\mathbf{u}_{b} \cdot \nabla H\right)+F_{w}\right]=0$,

where $\operatorname{curl} \tau$ is the vertical component of the wind stress curl at the ocean surface and $F^{w}$ is a measure of its uncertainty.

4. Stationary advective balance equations:

$\left(\mathbf{u} \cdot \nabla C_{n}\right)+w \frac{\partial C_{n}}{\partial z}-F_{n}=0$,
$1 \leq n \leq N: \theta, S, C_{3}, \ldots, C_{N}$,

where $N$ is the total number of measured properties (potential temperature and salinity, as well as other properties, measured along the section) and $F_{n}$ are unknown sources and sinks attributed to dynamical processes of smaller scales like turbulent mixing and to other phenomena of any nature (chemical reactions, biology, etc.).

5. The kinematic constraint on the net cross-section transport:

$\int v \mathrm{~d} \Omega-\left(F_{V}^{0}+F_{V}\right)=0$,

where $F_{V}^{0}=0$ is a prior estimate of the transport and $F_{V}$ its unknown error.

The model grid for property and density fields consists of two grid planes parallel to and equidistant from the section plane, where the measurements are actually taken. Grids for the other variables $\left(\mathbf{u}, w, F_{n}\right)$ are located on the section plane. Specifying the values of the control variables $C_{n}, \mathbf{u}_{b}, F_{w}$ and $F^{w}$, one may compute all the rest of the state vector components via Eqs. (A1-A5), and the value of its probability density using Eq. (1). The assimilation algorithm performs a search for the most probable state in the space of control variables.

To drive the assimilation pattern away from states with unstable density profiles a term $J_{e}$ is added to the cost function:

$J=J_{0}+J_{e} \equiv J_{0}+\int \exp \left(\frac{1}{\rho_{z}^{c}} \frac{\partial \rho}{\partial z}\right) \mathrm{d} \Omega$

where $\rho_{z}^{c}$, is a critical density gradient chosen to be $1 \times 10^{-15} \mathrm{~kg} / \mathrm{m}^{4} . J_{e}$ makes no contribution to the cost function for stratifications far from instability, but in the vicinity of unstable states, it helps to push the solution away. $J_{e}$ has no statistical interpretation and plays the role of a range constraint in continuous form, e.g. Schröter and Wunsch (1986).

Acknowledgements. We are grateful to Gerd Rohardt who was responsible for the mooring and CTD work during the cruises and the data processing. Dr. L. Goeyens, Dr. L. Gordon, Dr. M. Hoppema, K.-U. Richter and A. Wisotzki kindly provided the oxygen, nutrient and carbon dioxide data. The optimization routines were kindly provided by J. C. Gilbert from Institut National de Recherche en Informatique et en Automatique, BP105, F-78153 Le Chesnay, France. The research was supported by in part by Deutsche Forschungsgemeinschaft (SBF 318), AWI contribution number 1098 .

Topical Editor D.Y. Webb thanks C. Wunsch and H. Mercier for their help in evaluating this paper. 


\section{References}

Fahrbach, E., Cruise report ANT IX/2, In: The expeditions ANTARKTIS IX/1-4 of the Research Vessel "Polarstern" in 1990/91, Bathmann, U., M. Schulz-Baldes, E. Fahrbach, V. Smetacek and H.-W. Hubberten (Eds.), Ber. Polarforsch., 100, 31-94, 1992.

Fahrbach, E., Cruise report ANT X/7, In: The expeditions ANTARKTIS X/6-8 of the Research Vessel "Polarstern" in 1992/1993, in Eds. Bathmann U., V. Smetacek, H. De Baar, E. Fahrbach and G. Krause. Ber. Polarforsch. 135, 127-197, 1994.

Fahrbach, E., M. Knoche, and G. Rohardt, An estimate of water mass transformation in the southern Weddell Sea, Mar. chem., 35, 25-44, 1991.

Fahrbach, E., G. Rohardt, M. Schröder, and V. Strass, Transport and structure of the Weddell Gyre, Ann. Geophysicae., 12, 840855, 1994.

Fahrbach, E., G. Rohardt, N. Scheele, M. Schröder, V. Strass, and A. Wisotzki, Formation and discharge of deep and bottom water in the Northwestern Weddell Sea, J. Mar. Res., 53 (4), 515-538, 1995.

Augstein, E., Bagryantsev N., and H. W. Schenke, Eds., The expedition ANTARKTIS VIII/1-2, 1989, with the Winter Weddell Gyre study of the Research Vessels "Polarstern" and "Akademik Fedorov", Ber. Polarforsch., 84, 134pp, 1991.

Carmack, E. C., and T. D. Forster, On the flow of water out of the Weddell Sea, Deep-Sea Res., 22, 711-724, 1975.

Fofonoff, N. P., and R. C. Millard Jr., Algorithms for computation of fundamental properties of seawater, UNESCO Tech. Pap. in Mar. Sci., 44, 53 pp., 1983.

Gilbert, J. C. and C. Lemaréchal, Some numerical experiments with variable-storage quasi-Newton algorithms, Math. Progr., 45, 407-435, 1989.

Gill, A. E., Circulation and bottom water formation in the Weddell Sea, Deep-Sea Res., 20, 11-40, 1973.

Glowienka-Hense, R., A. Hense, and C. Völker, ECMWF versus Hellerman and Rosenstein wind stress climatology of the Southern Ocean, Antarct. Sci., 4 (1), 111-117, 1992.

Goeyens, L., E. Fahrbach, T. Behmann, H.-H. Hinrichsen, J. Krest, A. Ross, and A. Wisotzki, The summer Weddell Gyre study, Data report No 1, Preliminary Physiochemical Data, Ber. Fachbereich Physik AWI, 12, 54 pp., 1991.

Gordon, A. L., and B. A. Huber, Southern Ocean winter mixed layer, J. Geopys. Res., 95, 11 655-11 672, 1990.
Gordon, A. L., D. G. Martinson, and H. W. Taylor, The winddriven circulation in the Weddell-Enderby Basin, Deep-Sea Res., 28, 151-163, 1981.

Ivanov, Y. A., Antarctic bottom water formation, 21-27, In: Largescale and synoptic variability of oceanic fields, $167 \mathrm{pp}$., Nauka, Moscow, 1981 (in Russian).

Le Dimet, F.-X., and O. Talagrand, Variational algorithms for analysis and assimilation of meteorological observations: theoretical aspects, Tellus, 38a, 97-110, 1986.

Nechaev, D. A., and M. I. Yaremchuk, Application of the adjoint technique to processing of a standard section dataset: World Ocean Circulation Experiment section 4 along $67^{\circ} \mathrm{S}$ in the Pacific Ocean, J. Geophys. Res., 100, 865-879, 1995.

Nechaev, D. A., M. I. Yaremchuk and J. Schröter, A steady state inverse model of the large scale circulation of the Weddell Sea, Int. WOCE Newlett, 21, 11-14, 1995.

Needler, G. T., The absolute velocity as a function of conserved measurable quantities, Prog. Oceanogr., 14, 421-429, 1985.

Olbers, D., and C. Wübber, The role of wind and buoyancy forcing of the Antarctic Circumpolar current, In: Strategies for Future Climate Research, Ed. M. Latif., Max-Planck Institute für Meteorologie, Hamburg, 161-191, 1991.

Orsi, A. H., W. D. Nowlin, and T. Willworth III, On the circulation and stratification of the Weddell Gyre, Deep-Sea Res., 40, 169203, 1993.

Schröter, J., and C. Wunsch, Solution of non-linear finite-difference open ocean models by optimization method with sensitivity and observational strategy analysis, J. Phys. Oceanogr., 16, 1855$1875,1986$.

Stommel, H., and F. Schott, The $\beta$-spiral and the determination of the absolute velocity field from hydrographic data, Deep Sea Res., 24, 325-329, 1977.

Thacker, W. C., On the role of Hessian matrix in fitting models to data, J. Geophys. Res., 94, 6177-6196, 1989.

Weiss, R. F., H. G. Östlund, and H. Craig, Geochemical studies of the Weddell Sea, Deep-Sea Res., 26, 1093-1120, 1979.

Wunsch, C., The North Atlantic circulation west of $50^{\circ} \mathrm{W}$ determined by inverse methods, Rev. Geophys., 16, 583-620, 1978.

Wunsch, C., Dynamically consistent hydrography and absolute velocity in the eastern North Atlantic ocean, J.Geophys. Res., 99, 14 071-14 090, 1994. 